\title{
Formation and structure of the microemulsion phase in two-dimensional ternary $\mathrm{AB}+\mathrm{A}+\mathrm{B}$ polymeric emulsions
}

\author{
Dominik Düchs and Friederike Schmid \\ Fakultät für Physik, Universität Bielefeld, \\ Universitätsstr. 25, 33615 Bielefeld, Germany
}

\begin{abstract}
We present an analysis of the structure of the fluctuation-induced microemulsion phase in a ternary blend of balanced $\mathrm{AB}$ diblock copolymers with equal amounts of $\mathrm{A}$ and $\mathrm{B}$ homopolymers. To this end, graphical analysis methods are employed to characterize two-dimensional configuration snapshots obtained with the recently introduced Field-Theoretic Monte Carlo (FTMC) method. We find that a microemulsion forms when the mean curvature diameter of the lamellar phase coincides roughly with the periodicity of the lamellar phase. Further, we provide evidence to the effect of a subclassification of the microemulsion into a genuine and a defect-driven region.
\end{abstract}

\section{INTRODUCTION}

Microemulsions arise when two immiscible substances are compatibilized by the use of a surfactant lowering the interfacial tension between the incompatible components dramatically. Originally introduced for mixtures of oil, water, and a surfactant, the term microemulsion applies equally to polymeric blends. Microemulsions appear in two forms: droplet and bicontinuous. Here we consider the bicontinuous case, which is observed in symmetric systems with balanced surfactants. In polymeric emulsions, the compatibilizing copolymer is usually the most expensive ingredient. A good theoretical understanding of these system is therefore desirable from an application point of view, as well. Applications often depend crucially on the morphology of the blend, and bicontinuity is a particularly useful property, e. g., for improved stiffness or conductivity.

Traditionally, there have been three distinct coarsegrain approaches to the theoretical study of selfassembling amphiphilic systems 1]: (a) Microscopic approaches 2], often built around lattice-gas or latticeBoltzmann simulations 3, 4, 5, 6, 7, 8]; also by dissipative particle dynamics 9, 10, 11] or by standard Monte Carlo or molecular dynamics methods 12, 13, 14, 15, 16, 17, 18, 19]. (b) Ginzburg-Landau models 20, 21, 22, 23, 24, 25], leading to a much simpler description by means of only a few order parameters and coefficients which can be obtained from experiment. Polymeric blends have been described very successfully with a particular continuous density functional approach usually denoted selfconsistent field theory (SCFT) 27, 28, 29]. The drawback of this method is that it employs a mean-field approximation and therefore neglects the very cause for the existence of microemulsions in these systems. (c) Membrane approaches for systems in which the solubility of the amphiphiles is extremely low, such that they can form membranes by themselves 26]. This case is not considered here.
As mentioned above, the addition of a compatibilizing agent, here the $\mathrm{AB}$ copolymer, is essential to the formation of mesoscopically ordered phases in a pure A and B homopolymer mixture. The melts would otherwise phase-separate macroscopically below some relatively high temperature, which is typically above room temperature. Microemulsions, although by definition part of the disordered regime, do have a finite (mesoscopic) preferential length scale, and thus their existence depends on the presence of a compatibilizer, as well. Apart from the microscopic correlation length, $\xi$, which is the characteristic decay length of the correlation function, a second length scale, $q^{-1}$, which describes the wavelength of oscillations in the correlation function, comes into play. The disorder line is now defined as the locus where $q$ just vanishes. The onset of the microemulsion regime, on the other hand, is defined by the Lifshitz line, which is the locus where the peak in the structure factor is just found at nonzero wavevector $q^{*}$. A microemulsion is thus disordered but not structureless 1, 48.

In a recent paper, we introduced a new approach to incorporating the effect of thermal fluctuations in field theories of polymer melts: the Field-Theoretic Monte Carlo (FTMC) method [30]. It is an extension of the earlier mentioned SCFT method. The system under investigation was the ternary model system consisting of symmetric $\mathrm{AB}$ copolymers as well as $\mathrm{A}$ and $\mathrm{B}$ homopolymers, the homopolymers being $\alpha=0.2$ times as long as the copolymers. This system had previously been the object of a series of experiments conducted by Bates et al. 31, 32, 33. Mean-field calculations of its phase diagram predict a Lifshitz critical point where the disordered, lamellar, and phase-separated regions meet 34, 35]. However, it could be demonstrated both in these experiments as well as in the FTMC simulations that the Lifshitz point is destroyed by thermal fluctuations and a channel of bicontinuous microemulsion $(B \mu E)$ emerges in between the lamellar and phase-separated regions. Whereas the aim of that paper, Ref. 30, was mainly to establish the validity of the FTMC method and to compare it with a related com- 
plex Langevin method 36, 37, 38], we here want to examine more closely the formation and structure of the microemulsion phase.

Neither the disorder line nor the total monomer Lifshitz line correspond to any thermodynamic transition. Recently, Morkved et al. 's 39] have found that neither line correlates well with the transition from a fully disordered mixture at higher temperatures to a well-developed $B \mu E$. Nevertheless, they found that dynamic light scattering provided a clear signal for this transition, which they proposed to be the homopolymer/homopolymer Lifshitz line originally introduced by Holyst and Schick [40]. In a similar experimental system, Schwahn et al. [41] found evidence for the existence of three different regimes in the disordered phase, a "disordered blend", a "microemulsion", and a "disordered copolymer" region. The three regimes differ from each other by the value of the peak wavevector $q^{*}$ in the structure factor - zero in the disordered blend, large in the disordered copolymer, intermediate in the microemulsion. On the theoretical side, Holyst and Przybylski 42 performed Monte Carlo simulations of a Ginzburg-Landau model for the lamellar phase in copolymers and showed that topological fluctuations change the monotonic decay of the off-specular scattering intensity, introducing the topological Lifshitz line. One might suspect that such fluctuations affect the characteristic lamellar distance to higher values. Experiments 43 ] as well as simulations 44 indeed reveal that the peak wavevector $q^{*}$ is $\sim 15-20 \%$ lower at the order-disorder transition than predicted by SCFT. However, this effect can also be explained by local chain stretching [44, 45].

With our approach, we can separate the effect of fluctuations from that of local chain rearrangements. It combines the advantages of SCFT, which accounts in full for the chain connectivity but neglects fluctuations, with those of Ginzburg-Landau simulations, which include fluctuations but make approximations for the intrachain correlations. Our results are qualitatively similar to those of Morkved et al. and Schwahn et al. Our main result in this paper is the subclassification of the microemulsion region into a disordered and a genuine regime. For the transition from the lamellar to this disordered microemulsion phase we do not find a markedly lowered $q^{*}$. In the genuine regime, $q^{*}$ differs from the mean field value. Indeed, this is our criterion for the distinction of the two. It should be interesting to examine to what extent, if any, our subclassification corresponds to Morkved et al. 's [39] distinction of poor and good microemulsions, or to Schwahn et al. 's distinction of disordered copolymers and microemulsions.

In the present work, we have used parallel Cray architectures to simulate $\mathrm{AB}+\mathrm{A}+\mathrm{B}$ melts on two-dimensional lattices of size $48 \times 48$. The high demands on computing power have so far limited us to two dimensions. It will be straightforward to adapt the method to three dimensions once this becomes feasible from a computational point of view. This paper is organized as follows. In Section II, we shall briefly outline the theoretical model underlying FTMC. For a more detailed description, we refer the interested reader to our earlier paper 30. Section III contains the results and discussion. In this context, our evidence is presented to the effect of a subclassification of the microemulsion. We conclude in Section IV with a summary.

\section{THE FIELD-THEORETIC MODEL}

In this section, we briefly present the model underlying FTMC for the ternary system under consideration. For details, see Ref. 30 . We study a mixture of $n_{A}$ homopolymers of type $\mathrm{A}, n_{B}$ homopolymers of type $\mathrm{B}$, and $n_{A B}$ symmetric block copolymers in a volume $V$. The polymerization index of the copolymer is denoted by $N$, and the corresponding quantities for the homopolymers are denoted as by $N_{A}=N_{B}=\alpha N$. We consider the case of a symmetric copolymer, wherein the fraction of A monomers in the copolymer is $f=1 / 2$. We restrict our attention to the concentration isopleth, where the homopolymers have equal volume fractions $\phi_{H A}=\phi_{H B}=\phi_{H} / 2$. The monomeric volumes of both $\mathrm{A}$ and $\mathrm{B}$ segments are assumed to be identically equal to $1 / \rho_{0}$. On this model we impose an incompressibility constraint. We model the effective interactions between segments as Flory-Huggins local contact interactions and use a Gaussian chain model, which corresponds to perfectly flexible polymers. With these assumptions, the canonical partition function of the system can be written as

$$
\mathcal{Z}_{C} \propto \int_{\infty} D W_{-} \int_{i \infty} D W_{+} \exp \left[-H_{C}\left(W_{-}, W_{+}\right)\right]
$$

with

$$
\begin{gathered}
H_{C}\left(W_{-}, W_{+}\right)=C\left[\frac{1}{\chi N} \int d \mathbf{r} W_{-}^{2}-\int d \mathbf{r} W_{+}\right. \\
\left.-V\left(1-\phi_{H}\right) \ln Q_{A B}-\frac{V \phi_{H}}{2 \alpha} \ln Q_{A}-\frac{V \phi_{H}}{2 \alpha} \ln Q_{B}\right] \\
C=\frac{\rho_{0}}{N} R_{g}^{d}
\end{gathered}
$$

In this paper, all lengths are expressed in units of the unperturbed radius of gyration, $R_{g}=b(N /(2 d))^{1 / 2}$, where $d$ is the space dimension. The parameter $C$ in the above equations, which occurs as a global prefactor to $H_{C}$, acts as a Ginzburg parameter such that in the limit $C \rightarrow \infty$ the partition function (11) is reduced to its saddle point and the mean-field solution becomes exact. In Eq. (2), 
$Q_{A}, Q_{B}$, and $Q_{A B}$ denote the single chain partition functions for the $\mathrm{A}, \mathrm{B}$, and $\mathrm{AB}$ chains, respectively, in the potential fields $W_{-}(\mathbf{r})$ and $W_{+}(\mathbf{r})$. Note that $W_{-}$is conjugate to the difference in $\mathrm{A}$ and $\mathrm{B}$ densities, $\hat{m}$, and $W_{+}$ to the total density, $\hat{\phi}$. Moreover, $W_{-}$is real, whereas $W_{+}$is imaginary thereby rendering $H_{C}$ complex. We here employ a partial saddle point approximation in $W_{+}$, which reduces $H_{C}$ to real values.

The single chain partition functions can be expressed in terms of the Feynman-Kac formulae [28] as:

$$
Q_{i}=\int d \mathbf{r} q_{i}\left(\mathbf{r}, \nu_{i}\right)
$$

where the propagators $q_{i}$ satisfy diffusion equations. From these propagators, we can calculate density operators, $\bar{\phi}_{A}$ and $\bar{\phi}_{B}$, from

$$
\begin{aligned}
\bar{\phi}_{A}(\mathbf{r})= & \frac{V\left(1-\phi_{H}\right)}{Q_{A B}} \int_{0}^{f} d s q_{A B}(\mathbf{r}, s) q_{A B}^{\dagger}(\mathbf{r}, 1-s) \\
& +\frac{V \phi_{H}}{2 \alpha Q_{A}} \int_{0}^{\alpha} d s q_{A}(\mathbf{r}, s) q_{A}(\mathbf{r}, \alpha-s),
\end{aligned}
$$

and a similar equation for the $\bar{\phi}_{B}$. The densities which correspond to the experimentally measurable quantities are the averages over $\bar{\phi}_{A, B}$, i. e., $\phi_{A, B}=\left\langle\bar{\phi}_{A, B}\right\rangle$. For the calculations in the present work, however, we have treated $\bar{\phi}_{A}$ and $\bar{\phi}_{B}$ as instantaneous densities. We then calculated time averages over parameters calculated on them. Strictly speaking, the quantities $\bar{\phi}_{A, B}$ are visualizations of the spatial distributions of fields. The time averages so obtained should nevertheless reflect the essential structural properties of the system.

For a detailed description of the FTMC method used to obtain the configuration series that serve as the input data in this work, see Ref. 30 .

\section{RESULTS AND DISCUSSION}

Fig. \shows the fluctuation-corrected phase diagram of the ternary melt, as obtained in Ref. [30], at dimensionless polymer number density $C=50$, which is an intermediate value and has been used throughout this work. Compared with the corresponding mean-field diagram, one discerns (a) a shift in the order-disorder transition to higher segregation strengths, $\chi N$, (b) a slight shift in the same direction of the transition between the disordered and phase-separated regions, and (c) the emergence of a cusp-like region of a microemulsion phase. Note that this diagram was calculated in two dimensions, due to numerical constraints. Likewise, the simulations carried out in the present work were in two dimensions only. While a full three-dimensional analysis will likely yield a more

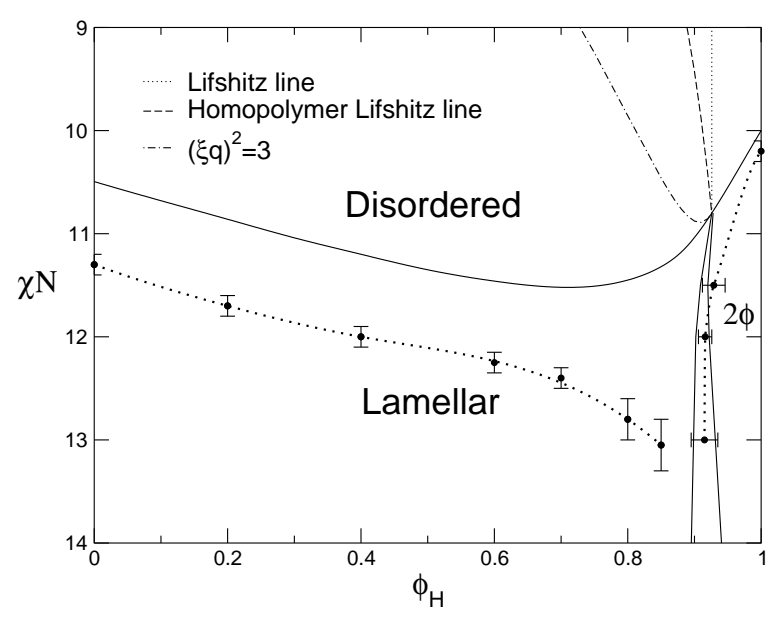

FIG. 1: Phase diagram of the ternary $\mathrm{A}+\mathrm{B}+\mathrm{AB}$ blend $(\alpha=$ 0.2 ). The solid lines show the mean-field phase diagram, which features a three-phase $(\mathrm{L}+\mathrm{A}+\mathrm{B})$ coexistence region reaching up to the Lifshitz point. The circles give locations of fluctuation corrected phase boundaries at $C=50$ (from Ref. [30]). The thick dotted lines are guides for the eye. The orderdisorder transition is weakly first-order, but the coexistence region is too small to be displayed. The thin lines in the disordered region indicated the total monomer Lifshitz line (dotted), the homopolymer-homopolymer Lifshitz line (dashed), and the line of $(q \xi)^{2}=3$ (dashed-dotted), as obtained from mean-field theory.

narrow microemulsion "channel" than that shown in Fig. [1 the good qualitative agreement with experiment indicates that essential aspects of the phase behavior are captured in two dimensions already. Thus it is justified to proceed with further analysis.

In addition to these results taken from our earlier paper, Ref. 30, Fig. 1 also displays various Lifshitz lines calculated within the mean-field approximation. The total monomer Lifshitz line is found at the homopolymer volume fraction 46 ]

$$
\Phi_{H}=\frac{1}{1+2 \alpha^{2}},
$$

and is independent of the incompatibility parameter $\chi N$. The homopolymer-homopolymer Lifshitz line, on the other hand, is independent of $\alpha$, and determined by the equation

$$
\chi N=\sqrt{\frac{8}{\Phi_{H}\left(1-\Phi_{H}\right)}} .
$$

It was obtained by generalizing the calculation of Holyst and Schick 40 for homopolymer/copolymer length ratio $\alpha=1$ to arbitrary $\alpha$. Note that in contrast to the case $\alpha=1$ studied by Holyst and Schick, the homopolymerhomopolymer Lifshitz line is rather close to the total 
monomer Lifshitz line at $\alpha=0.2$. This is because short homopolymers swell the copolymer blocks, whereas the longer homopolymers in systems with $\alpha=1$ are expelled from the copolymer rich regions.

Finally, Fig. 10 also shows the line $(q \xi)^{2}=3$, which distinguishes between microemulsions with weak and strong ordering tendency in confined geometries [47]. Here $\xi$ is the correlation length and $q$ the wavevector $q$ of oscillations in the correlation function. The line $(q \xi)^{2}=3$ was calculated by expanding the inverse mean-field structure factor $S(q)^{-1}$ up to fourth order in $q$, i. e., approximating $S(q)$ by the Teubner-Strey form [48],

$$
S(q)=\frac{1}{\omega+g q^{2}+c q^{4}}
$$

and then determining the value of $\chi N$ where $g^{2} / \omega c=$ 1 47.

We start by examining the structure factor of our twodimensional melts. It can be calculated from the Fourier transform of the density correlation function, which in turn can be obtained from the fluctuating $W_{A}(\mathbf{r}) \equiv$ $W_{+}(\mathbf{r})+W_{-}(\mathbf{r})$ field via a formula derived in Ref. 37 . Thus, the structure factor $S(\mathbf{q})$ is the Fourier transform of

$$
\frac{1}{V} \int \mathrm{d} \mathbf{r}_{0}\left[\left\langle\hat{\rho}\left(\mathbf{r}_{0}\right) \hat{\rho}\left(\mathbf{r}_{0}+\mathbf{r}\right)\right\rangle-\left\langle\hat{\rho}\left(\mathbf{r}_{0}\right)\right\rangle\left\langle\hat{\rho}\left(\mathbf{r}_{0}+\mathbf{r}\right)\right\rangle\right],
$$

with

$$
\begin{aligned}
& \left\langle\hat{\rho}(\mathbf{r}) \hat{\rho}\left(\mathbf{r}^{\prime}\right)\right\rangle-\langle\hat{\rho}(\mathbf{r})\rangle\left\langle\hat{\rho}\left(\mathbf{r}^{\prime}\right)\right\rangle=\frac{2 C}{\chi N} \delta\left(\mathbf{r}-\mathbf{r}^{\prime}\right) \\
& -\frac{4 C^{2}}{(\chi N)^{2}}\left[\left\langle W_{A}(\mathbf{r}) W_{A}\left(\mathbf{r}^{\prime}\right)\right\rangle-\left\langle W_{A}(\mathbf{r})\right\rangle\left\langle W_{A}\left(\mathbf{r}^{\prime}\right)\right\rangle\right] .
\end{aligned}
$$

In Fig. 2] structure factors for lamellar phases and strongly and weakly structured microemulsions are shown. The lamellar structure factor ( Fig. 2]a) features the hallmark double peak of a striped pattern. Fig. 2 b) illustrates that in a microemulsion, anisotropy is lost yet a preferential length scale does exist, as evidenced by a ring-shaped region of maxima. As we progress deeper into the disordered phase at lower $\chi N$, the ring becomes less pronounced, until it is almost indistinguishable from a a shapeless low-amplitude noise ( Fig. 2] c).

The above procedure to calculate the structure factor of a configuration is quite expensive from a computational standpoint as it involves integrations over the entire course of a simulation. However, if we are to analyze geometrical patterns, it is not necessary to use the fullfledged structure factor. We can see the characteristic features of a configuration from the Fourier transform of its density distribution, as well. In this work, we have therefore used time averages of quantities derived from the Fourier transform of $\bar{\phi}_{A}$ in analyzing morphological properties.

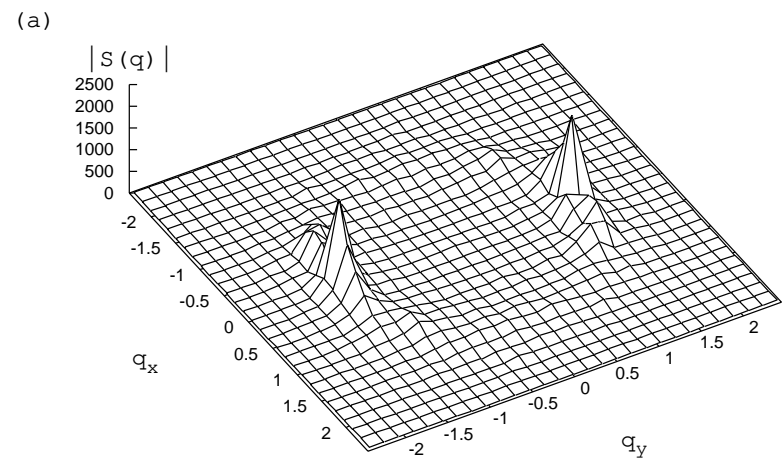

(b)

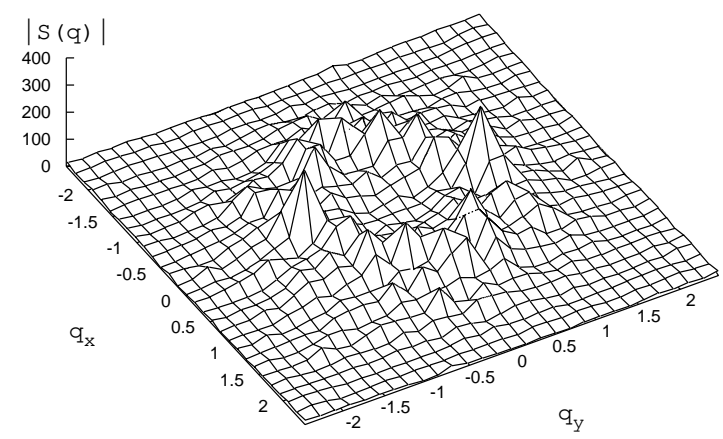

(c)

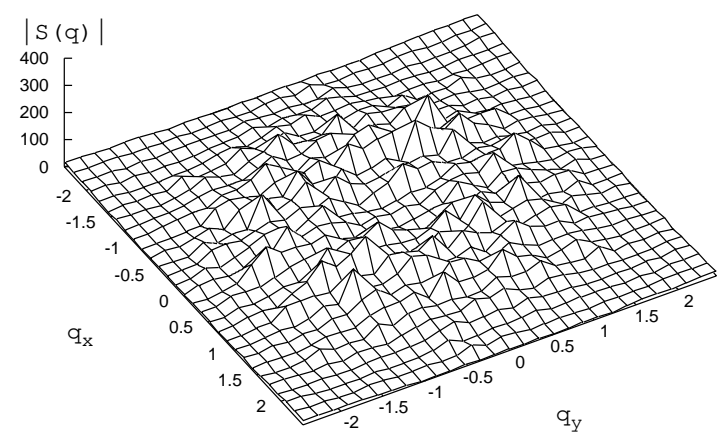

FIG. 2: Structure factors at $C=50$ (a) Lamellar phase at $\phi_{H}=0.7$ and $\chi N=12.5$. (b) Strongly structured microemulsion at $\phi_{H}=0.82$ and $\chi N=12.5$. (c) Weakly structured microemulsion at $\phi_{H}=0.82$ and $\chi N=10$. 
As a starting point, we need to find the preferential length scale of a density distribution $\bar{\phi}_{A}(\mathbf{r})$. This is equivalent to finding the preferential wave vector of its twodimensional Fourier transform, $\mathrm{F}(\mathbf{q})$. To this end, we use the square averaged over all angles, defining

$$
F_{0}(q):=\frac{1}{2 \pi} \int_{0}^{2 \pi} d \phi|F(\mathbf{q})|^{2},
$$

which is now one-dimensional.
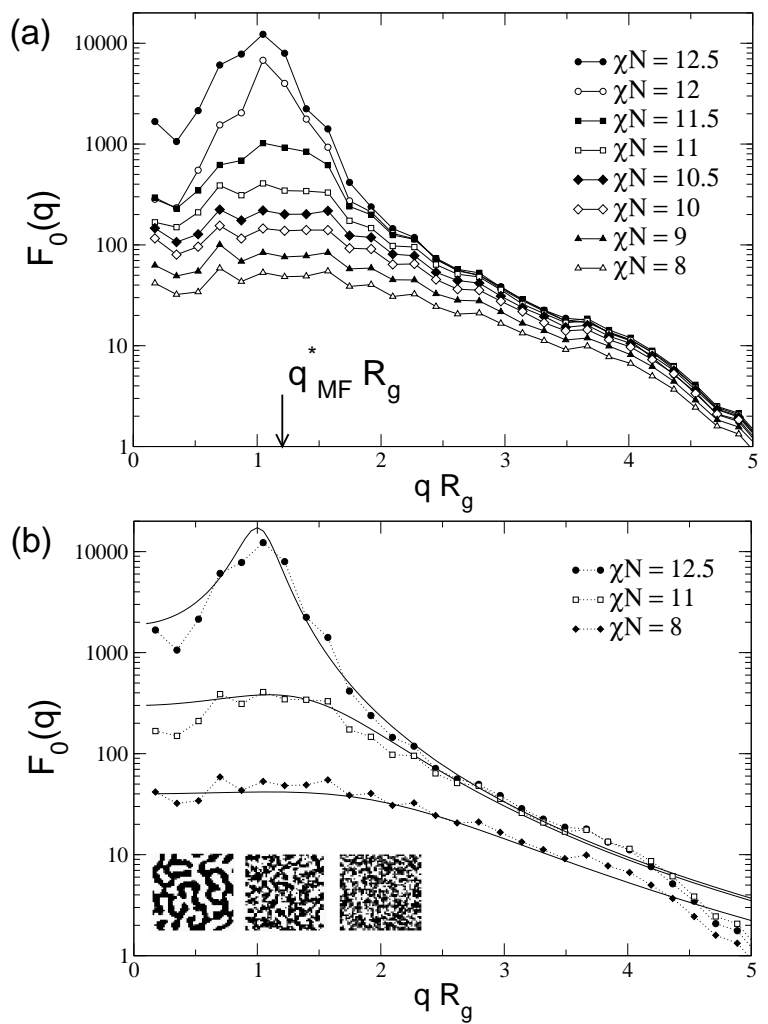

FIG. 3: (a) Equilibrated averages of $F_{0}(q)$ at $C=50$, $\Phi_{H}=0.82$, and different values of $\chi N$. The arrow indicates the mean-field location $q_{M F}^{*}$ of the maximum in the structure factor in the disordered phase. (b) Teubner-Strey fits to $F_{0}(q)$ for selected values of $\chi N$. The inset shows snapshots of $\phi_{A}$ for the same $\chi N$ values $(12.5,11$, and 8 from left to right). A lattice point is painted black ("A") if $0.51 \leq \bar{\phi}_{A} \leq 1$, grey if $0.49 \leq \bar{\phi}_{A}<0.51$, and and white ("B") if $0 \leq \bar{\phi}_{A}<0.0 .49$.

From Fig. 3] in which $F_{0}(q)$ is displayed for various $\chi N$ at $\phi_{H}=0.82$, it can be seen that $F_{0}(q)$ has a pronounced peak for $\chi N \lesssim 11$. Below $\chi N \sim 11$ the curves become markedly flatter at low $q$. Note that the mean-field transition between the disordered and the lamellar phase occurs at $\chi N=11.4$ (for $\Phi_{H}=0.82$ ). Above this point, the position of the (hardly discernible) maximum of $F_{0}(q)$ agrees well with its mean-field value,
$q_{M F}^{*} R_{g}=1.203$ for $\Phi_{H}=0.82$. In the more structured region of higher $\chi N$, the peak moves to lower values of $q$, reflecting the fact that the corresponding mean-field length scale, the lamellar distance, increases with increasing $\chi N$. Fig. 3 b) shows fits of selected curves to the Teubner-Strey form ( Eq. (8) 48]. The fits are reasonable at high and low values of $\chi N$, and less satisfactory in the vicinity of the mean-field transition.

The technicalities of calculating $F_{0}(q)$ require $q$ to be binned in a histogram and thus approximated by discrete values (in practice, by multiples of $2 \pi / L$, where $\mathrm{L}$ is the box length). In addition, Fig. [3illustrates that our data for $F_{0}(q)$ are strongly scattered, mainly due to the statistical error. This makes the $q$ coordinate of the maximum of $F_{0}(q)$ a poor candidate to be taken as the preferential wave vector. A smoother procedure is to use

$$
\bar{q}=\frac{\int \mathrm{d} q q F_{0}(q)}{\int \mathrm{d} q F_{0}(q)} .
$$

If $F_{0}(q)$ has a pronounced well-defined maximum at nonzero $q^{*}$ (e. $g ., \chi N \geq 11.5$ in Fig. 3), $\bar{q}$ roughly coincides with this maximum. Otherwise, it slightly overestimates $q^{*}$ ( e. g., $q_{0} R_{g}=1.3$ at $\chi N=11 ., q_{0} R_{g}=1.6$ at $\chi N=8)$. Now, the preferential length scale, $L_{0}$, is defined as

$$
L_{0}:=\frac{2 \pi}{\bar{q}}
$$

Further, we define the mean curvature diameter, $D_{C}$, of the boundaries of $\mathrm{A}$ and $\mathrm{B}$ microdomains in a blackand-white image similar to those in the inset of Fig. $3 \mathrm{~b}$ ), in which a lattice point is painted black ("A") if $0.5<\bar{\phi}_{A} \leq 1$, and white ("B") if $0 \leq \bar{\phi}_{A} \leq 0.5$ :

$$
D_{C}:=2\left[\frac{1}{L_{c}} \int \mathrm{d} s\left|\frac{\mathrm{d} \mathbf{t}}{\mathrm{d} s}\right|^{2}\right]^{-\frac{1}{2}} .
$$

$L_{c}$ is the sum of all contour lengths of the microdomain boundaries, and $\mathbf{t}$ is the tangent vector at a given coordinate $s$ along the contour. For a detailed explanation of the algorithm used to calculate $D_{C}$, see Appendix A. Note that the snapshots shown here also contain grey pixels, corresponding to a balance density, i.e., $0.49<\bar{\phi}_{A}<0.51$.

In Figs. 4 5 [ 6 configurational snapshots 49] and plots of equilibrated averages of $L_{0}$ and $D_{C}$ are displayed for various homopolymer volume fractions, $\phi_{H}$, at $\chi N=$ $12.5,12$, and 11.5. The deviation of $L_{0}$ from the corresponding mean-field values, $L_{M F}$, is shown in the inset. The Monte Carlo simulations were run up to approximately 1.5 million Monte Carlo steps, including equilibration times of up to a few 100,000 steps. A Monte 
(a)
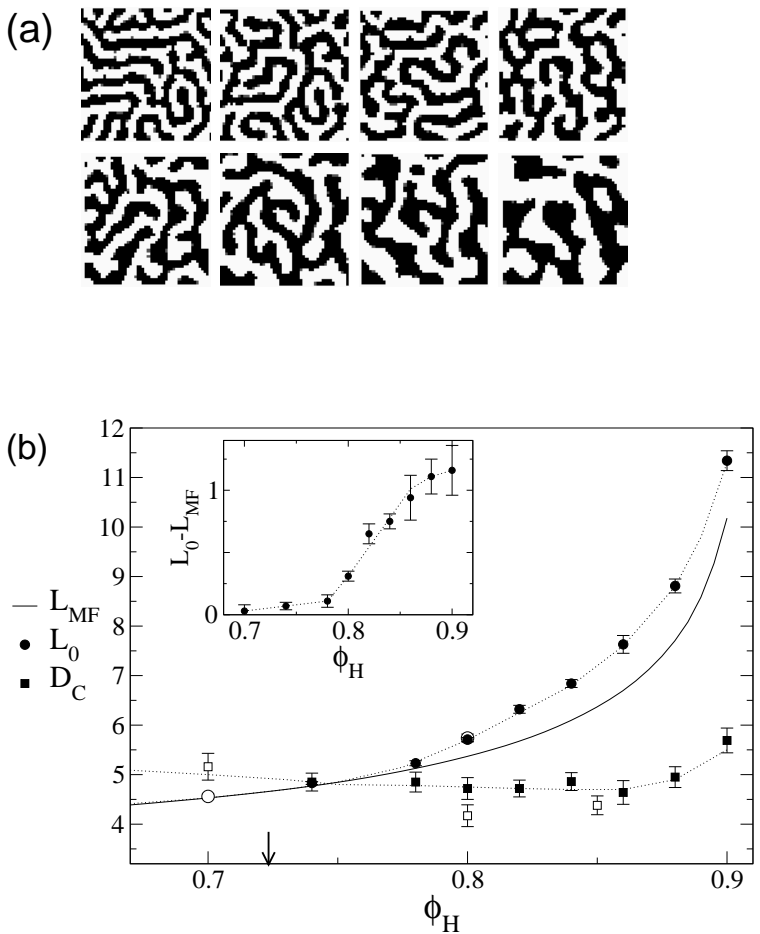

FIG. 4: Characteristic length scales at $\chi N=12.5, C=50$. (a) Snapshots at $\phi_{H}=0.74,0.78,0.8$, and 0.82 (first row from left), and $\phi_{H}=0.84,0.86,0.88$, and 0.9 (second row from left). (b) Preferential length scale, $L_{0}$ (circles), and curvature diameter, $D_{C}$ (squares), in units of $R_{g}$, vs. $\Phi_{H}$, averaged over the equilibrated parts of simulations on a $48 \times 48$ lattice. The solid line shows the mean-field lamellar distance $L_{M F}$ for comparison. Filled and empty symbols correspond disordered and lamellar initial conditions, respectively. The inset shows the difference of $L_{0}$ and $L_{M F}$.

Carlo step includes one attempted random local increment of $W_{-}$per pixel, within ranges that were chosen such that the Metropolis acceptance rate was $35 \%$. As a general rule, the more disordered the configurations, the shorter was the equilibration phase.

In all three cases, i.e., $\chi N=12.5,12$, and $11.5, D_{C}$ is in good approximation constant over an extended $\phi_{H}$ range (Figs. [4 5 and 6). This is plausible because the curvature is induced by the copolymers, which are located predominantly at the interfaces between microdomains. It should thus be independent of the amount of homopolymers. Nevertheless, $D_{C}$ gets somewhat smaller as $\chi N$ is decreased; the higher temperatures that correspond to lower $\chi N$ facilitate the bending of the microdomain interfaces.

At $\chi N=12.5$, coming from the lamellar phase at low $\phi_{H}$, the curvature radius $D_{C}$ becomes comparable in size to the preferential length scale, $L_{0}$, as well as the meanfield length scale, $L_{M F}$, around $\phi_{H} \approx 0.75$, which is when (a)
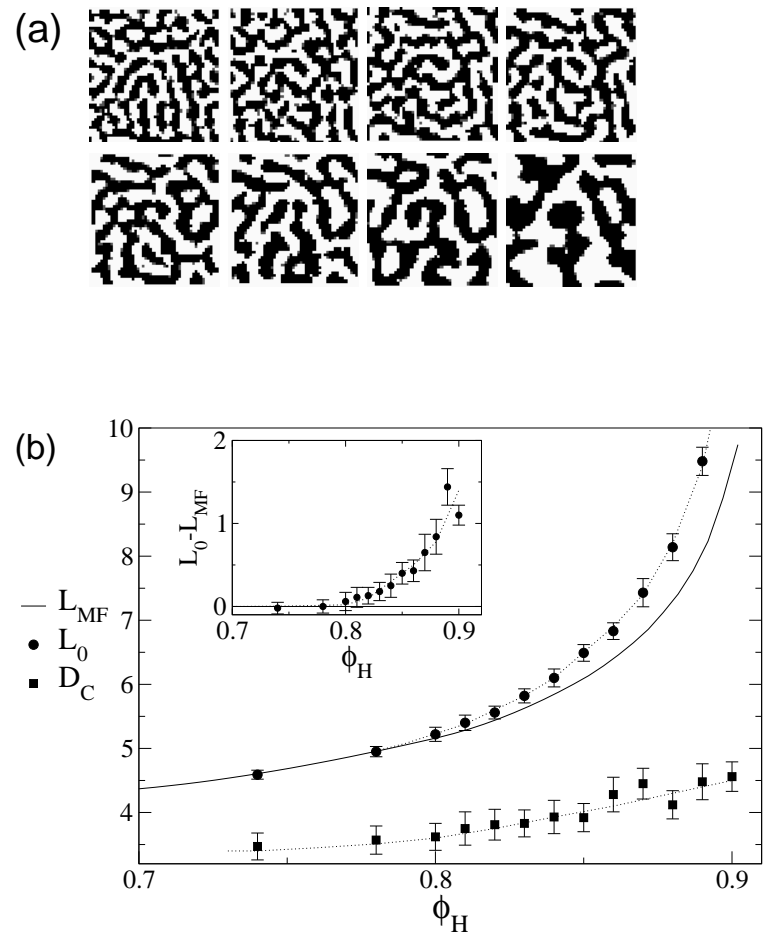

FIG. 5: Same as Fig. 4 for $\chi N=12$. As in Fig. 4 the snapshots in (a) correspond to $\phi_{H}=0.74,0.78,0.8$, and 0.82 (first row from left), and $\phi_{H}=0.84,0.86,0.88$, and 0.9 (second row from left).

the lamellae begin to break up. This is in good quantitative agreement with the location of the fluctuationcorrected order-disordered transition of Fig. 11. At $\chi N$ $=12$ and $11.5, D_{C}$ is well below both the mean-field periodicities, $L_{M F}$, and the preferential length scales, $L_{0}$, in the $\phi_{H}$ range examined: these configurations are in the disordered phase. We have thus identified the mechanism by which fluctuations generate the microemulsion.

The preferential length scale $L_{0}$ and the mean-field length scale $L_{M F}$ are identical within error bars at small homopolymer concentrations $\Phi_{H}$. Upon increasing $\Phi_{H}$ at $\chi N=12.5$ and 12 , one notes that $L_{0}$ begins to deviate from $L_{M F}$ at a certain $\phi_{H}$. This signals the departure from defect-driven behavior and the onset of a more genuine morphology within the microemulsion phase. The effect is stronger for $\chi N=12.5$ than for $\chi N=12$. At $\chi N=11.5$, it is barely noticeable anymore, and $L_{0}$ coincides within error bars with $L_{M F}$. However, the higher $\chi N$, the more smeared out $L_{0}$ becomes.

As pointed out already, a microemulsion is characterized by the existence of a preferential length scale within the disordered phase 1, 48]. In this sense, all configurations displayed in Figs. 4 [5] and [ are microemulsions. The previous result, however, suggests a more diversified classification into (a) a defect-driven region and 
(a)
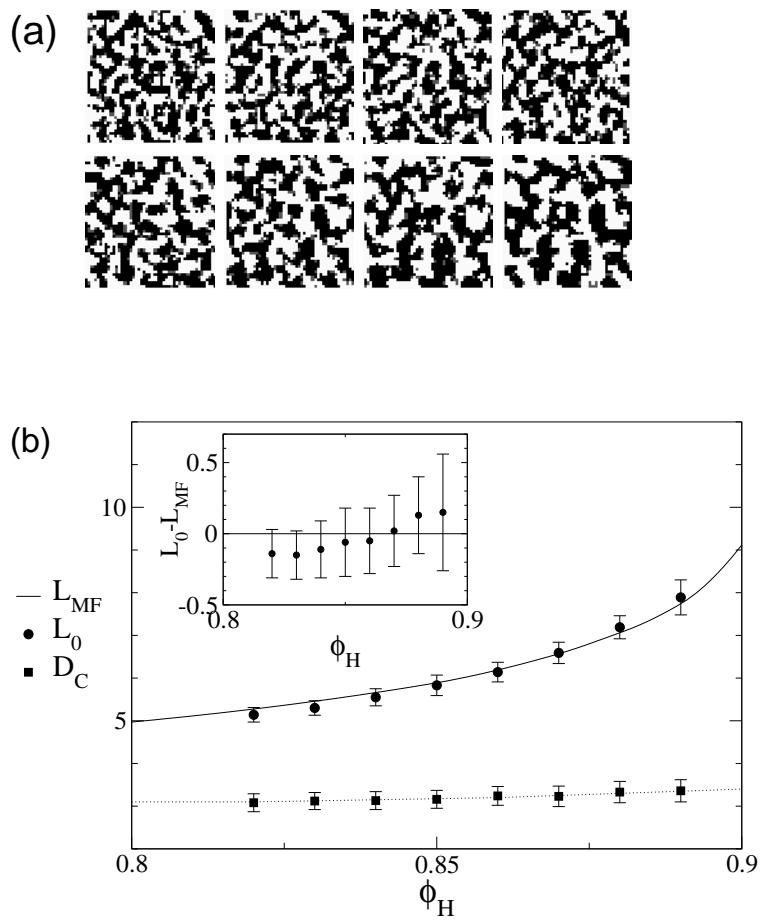

FIG. 6: Same as Fig. 4 for $\chi N=11.5$ The snapshots in (a) correspond to $\phi_{H}=0.82,0.83,0.84$, and 0.85 (first row from left), and $\phi_{H}=0.86,0.87,0.88$, and 0.89 (second row from left).

(b) a "genuine" microemulsion. We shall denote them $D \mu E$ and $G \mu E$, respectively. In the first region, the microemulsion retains the characteristic length scale of a fluctuation-free lamellar phase, and the main effect of the fluctuations is to introduce lamellar defects, which destroy the lamellar order. Typical configurations are the first snapshots (top row) in Figs. [4 and 5 a). In the second region, the interfaces themselves fluctuate, as can be observed in the images in the bottom row of Figs. 4 and 5 a). This causes an effective increase of the characteristic length scale (or decrease in the characteristic wavevector). Deeper in the disordered phase, at low $\chi N$, the distinction between lamellar defects and interfacial fluctuations is less obvious, one rather observes general structured, but unspecific disorder. In view of the fact that the characteristic wavevector coincides with the mean-field periodicity or, in the disordered phase, $q_{M F}^{*}$ ( Fig. 3), we classify it as $D \mu E$.

\section{SUMMARY AND CONCLUSION}

In this paper, we have investigated the structure of the disordered microemulsion phase in two-dimensional symmetrical ternary $\mathrm{AB}+\mathrm{A}+\mathrm{B}$ polymeric blends in dif-

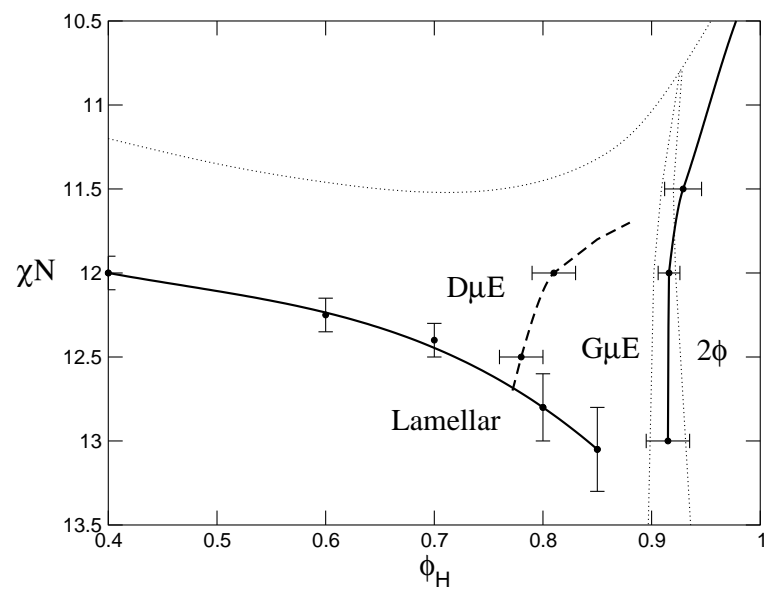

FIG. 7: Substructure of the microemulsion. $D \mu E$ : defectdriven, $G \mu E$ : genuine microemulsion morphology, $2 \phi$ : phaseseparated region. The solid lines show the fluctuationcorrected phase diagram of Fig. 11 the dotted lines the mean-field phase diagram, and the dashed line is just a guide for the eye.

ferent regions of the phase diagram. Not surprisingly, the structure of the microemulsion is strongest at parameters $\chi N$ and $\phi_{H}$ where the mean-field approximation would predict an ordered phase, $i$. e., where the disorder is brought about solely by fluctuations. We have corroborated our earlier result [30] regarding the mechanism that underlies the formation of the microemulsion phase in ternary $\mathrm{AB}+\mathrm{A}+\mathrm{B}$ polymeric blends: the lamellae break up when the curvature diameter of the microdomain boundaries becomes comparable to the periodicity of the lamellar phase 50 . We have further shown that the preferential length scale in the system deviates from its mean-field equivalent in a parameter subspace of the microemulsion only, demonstrating that the microemulsion region is divided into a defect-driven and a "genuine" part. In the resulting redrawing ( Fig. [7) of the phase diagram Fig. 10 we have indicated the approximate location of the conjectured transition between the two, as marked by the occurrence of nonzero values for $L_{0}-L_{M F}$ in Fig. 4 and 5 , Comparing Fig. 7 with the various mean-field Lifshitz lines indicated in Fig. 10 we find that both the total monomer Lifshitz line and the homopolymer-homopolymer Lifshitz line are far from the region of interest here. The numerical results presented in Fig. 3 confirm that this is still true for the true (fluctuation-corrected) total monomer Lifshitz line. We have not investigated the homopolymer-homopolymer structure factor. Since the short homopolymers in our system $(\alpha=0.2)$ swell the copolymers, the homopolymerhomopolymer Lifshitz line is not very far from the total monomer Lifshitz line.

It is possible that the $G \mu E$ phase coincides with the 
"microemulsion" $(\mu E)$ phase indicated in the experimental phase diagram of Schwahn et al. [41]. Strictly speaking, however, the two systems cannot be compared directly to each other, because our simulations were carried out in two dimensions only. We hope that threedimensional calculations will become feasible in the future.

We thank M. Matsen, V. Ganesan, and G. Fredrickson for fruitful discussions. This work was supported by the Deutsche Forschungsgemeinschaft (Germany). The simulations were carried out on the CRAY T3E of the NIC institute in Jülich.

\section{APPENDIX A: GRAPHICAL ANALYSIS}

In order to analyze configuration snapshots, it is often advantageous to take the $\bar{\phi}_{A}$ distribution and convert its continuous values (between 0 and 1) to black-and-white bitmaps with white pixels for $0 \leq \bar{\phi}_{A}(x, y)<0.5$, and black pixels for $0.5 \leq \bar{\phi}_{A}(x, y) \leq 1$. From these images, we can then extract the following parameters.

\section{Algorithm to calculate the circumference, $L_{c}$}

(1) Besides the original bitmap, $B_{1}$, define another bitmap, $B_{2}$, and paint it white, i.e., set all pixels to 0 .

(2) Copy all border pixels from $B_{1}$ to $B_{2}$. A border pixel is defined as one which is black (i.e., has a value of 1) and whose four nearest neighbors have at least one white pixel among them.

(3) Scan through $B_{2}$ from the upper left to the lower right corner and for each pixel check whether it is black. If so, proceed as follows:

(3a) If any pair of adjacent nearest-neighbor $(\mathrm{NN})$ and next-nearest-neighbor (NNN) pixels of the current pixel are both black, paint the $\mathrm{NN}$ pixel white. This is done to prevent ambiguities in the subsequent steps. In practice, however, this case is quite rare and barely changes the end result.

(3b) Check the distribution of black pixels among the nearest and next-nearest neighbors according to Fig. 8 and add the specified numbers to the circumference.

(4) Once the entire lattice has been scanned, divide the result by 2 to account for double counting in step (3b).

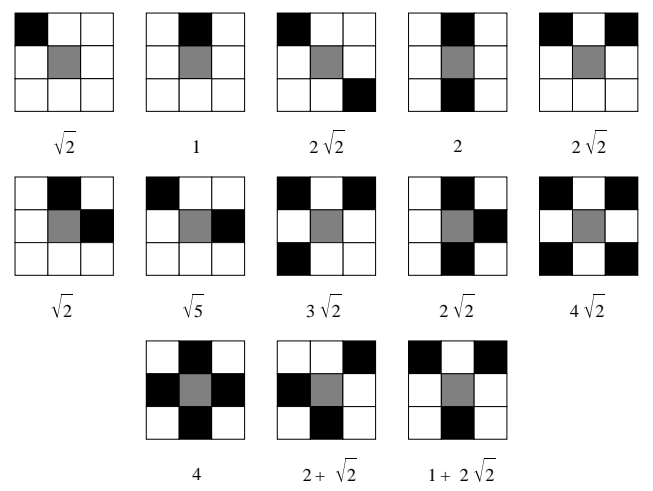

FIG. 8: Local contributions to the circumference. The current (black) pixel from step (3) here is painted grey. All diagrams are modulo $\pi / 2$ rotations.

\section{Algorithm to calculate the curvature diameter, $D_{C}$}

The curvature diameter of the (combined) circumference, $L_{c}$, of all black areas is defined as

$$
D_{C}:=2\left[\frac{1}{L_{c}} \int \mathrm{d} s\left|\frac{\mathrm{d} \mathbf{t}}{\mathrm{d} s}\right|^{2}\right]^{-\frac{1}{2}},
$$

with $\mathbf{t}$ the local (normalized) tangent vector. To calculate the quantity

$$
\frac{1}{L_{c}} \int \mathrm{d} s\left|\frac{\mathrm{d} \mathbf{t}}{\mathrm{d} s}\right|^{2}
$$

we pursue the following strategy:

(I.1) Make a border pixel bitmap as above.

(I.2) Scan the bitmap and stop when a black pixel is found.

(I.3) Start a data array, $\left(x_{j}^{i}, y_{j}^{i}\right)$, for the coordinates (numbered $j \geq 0$ ) of a new line, $i \geq 0$.

(I.4) Look for a (nearest or next-nearest) neighbor of the current pixel. In most cases, there will be only one possibility to continue the line. Otherwise, an arbitrary choice is made.

(I.5) Remove the current pixel from the bitmap. Make the neighboring pixel from (4) the current pixel.

(I.6) Repeat (I.4-5) until no neighbor is found for the current pixel. Then terminate that line.

(I.7) Repeat (I.2-6) until the bitmap has been cleared. 
(II.1) For each line $i,\left(x_{j}^{i}, y_{j}^{i}\right) \equiv\left(x_{j}, y_{j}\right)$, as determined in (I.1-7) that is longer than 3 points, set $j=1$.

(II.2) Calculate $|\mathrm{d} \mathbf{t} / \mathrm{d} s|^{2}$ according to

$$
\begin{aligned}
\mathrm{d} \mathbf{t}_{x}= & \frac{|\mathrm{d} \mathbf{t}|^{2}=\frac{\mathrm{d} \mathbf{t}_{x}^{2}+\mathrm{d} \mathbf{t}_{y}^{2}}{\mathrm{~d} s s^{2}}}{\sqrt{\left(x_{j+2}-x_{j}\right)^{2}+\left(y_{j+2}-y_{j}\right)^{2}}} \\
& -\frac{x_{j+1}-x_{j-1}}{\sqrt{\left(x_{j+1}-x_{j-1}\right)^{2}+\left(y_{j+1}-y_{j-1}\right)^{2}}}, \\
& -\frac{y_{j+2}-y_{j}}{\sqrt{\left(x_{j+2}-x_{j}\right)^{2}+\left(y_{j+2}-y_{j}\right)^{2}}} \\
& \mathrm{~d} x=\frac{y_{j+1}-y_{j-1}}{\sqrt{\left(x_{j+1}-x_{j-1}\right)^{2}+\left(y_{j+1}-y_{j-1}\right)^{2}}}, \\
\mathrm{~d} y=\frac{y_{j+2}-x_{j+1}+x_{j}-x_{j-1}}{2} &
\end{aligned}
$$

$$
\mathrm{d} s=\sqrt{\mathrm{d} x^{2}+\mathrm{d} y^{2}} .
$$

(II.3) Increase $j$. Repeat (II.2-3) until the end of the line is reached.

(II.4) Repeat (II.1-3) for all lines.

(II.5) Apply

$$
L_{c}=\int \mathrm{d} s .
$$

Note that this algorithm produces a slightly different (smaller) value for $L_{c}$ than the one presented in the last section. This because the length of each line is effectively truncated by three points. $L_{c}$ here acts only as a normalizing factor and for consistency should be calculated as indicated. If interested in $L_{c}$ itself, one should use the algorithm of the previous section.
[1] G. Gompper and M. Schick, Self-Assembling Amphiphilic Systems (Academic Press, London, New York, 1994).

[2] R. Rajagopalan, Curr. Opn. Coll. Interf. Sci. 6, 357 (2001).

[3] P. J. Love, P. V. Coveney, B. M. Boghosian, Phys. Rev. E 64, 021503 (2001).

[4] T. Sakai, Y. Chen, H. Ohashi, Phys. Rev. E 65, 031503 (2002).

[5] O. Theissen, G. Gompper, D. M. Kroll, Eur. Lett. 42, 419 (1998).

[6] M. Nekovee, P. V. Coveney, H. D. Chen, B. M. Boghosian, Phys. Rev. E 62, 8282 (2000).

[7] H. D. Chen, B. M. Boghosian, P. V. Coveney, M. Nekovee, P. Roy. Soc. London A 456, 2043 (2000).

[8] P. J. Love, M. Nekovee, P. V. Coveney, J. Chin, N. Gonzalez-Segredo, J. M. R. Martin, Comp. Phys. Comm. 153, 340 (2003).

[9] S. Jury, P. Bladon, M. Cates, S. Krishna, M. Hagen, N. Ruddock, P. Warren, Phys. Chem. Chem. Phys. 1, 2051 (1999).

[10] J. C. Shillcock, R. Lipowsky, J. Chem. Phys. 116, 5842 (2002).

[11] H. Guo, K. Kremer, J. Chem. Phys. 119, 9308 (2003).

[12] S. Karaborni, B. Smit, Curr. Opn. Coll. Interf. Sci. 1, 411 (1996).

[13] M. Müller, M. Schick, J. Chem. Phys. 105, 8885 (1996).

[14] C. A. Bearchell, D. M. Heyes, Phys. Chem. Chem. Phys. 3, 5255 (2001).

[15] D. Bedrov, G. D. Smith, K. F. Freed, J. Dudowicz, J.
Chem. Phys. 116, 4765 (2002).

[16] O. N. Vassiliev, M. W. Matsen, J. Chem. Phys. 118, 7700 (2003).

[17] A. Poncela, A. M. Rubio, J. J. Freire, J. Chem. Phys. 118, 425 (2003).

[18] C. Loison, M. Mareschal, K. Kremer, F. Schmid, J. Chem. Phys. 119, 13138 (2003).

[19] G. K. Bourov, A. Bhattacharya, J. Chem. Phys. 119, 9219 (2003).

[20] G. Gompper, M. Schick, Phys. Rev. Lett. 65, 1116 (1990).

[21] M. Olvera de la Cruz, Phys. Rev. Lett. 67, 85 (1991).

[22] R. Holyst, P. Oswald, J. Chem. Phys. 109, 11051 (1998).

[23] U. S. Schwarz, G. Gompper, Phys. Rev. E 59, 5528 (1999).

[24] F. Clarysse, C. J. Boulter, Physica A 278, 356 (2000).

[25] T. Schilling, G. Gompper, J. Chem. Phys. 117, 7284 (2002).

[26] G. Gompper, D. M. Kroll. J. Phys.: Cond. matt. 6, 8795 (1997).

[27] S. F. Edwards, Proc. Phys. Soc. 85, 613 (1965).

[28] E. Helfand, Y. Tagami, J. Polym. Sci. B 9741 (1971); E. Helfand, J. Chem. Phys. 56, 3592 (1972); E. Helfand, J. Chem. Phys. 62, 999 (1975)

[29] F. Schmid, J. Phys.: Cond. Mat. 10, 8105 (1998).

[30] D. Düchs, V. Ganesan, G. H. Fredrickson, and F. Schmid, Macromolecules 36, 9237 (2003).

[31] F. S. Bates, W. W. Maurer, P. M. Lipic, M. A. Hillmyer, K. Almdal, K. Mortensen, G. H. Fredrickson, and T. P. 
Lodge, Phys. Rev. Lett. 79, 849 (1997);

[32] T. L. Morkved, B. R. Chapman, F. S. Bates, T. P. Lodge, P. Stepanek, K. Almdal, Faraday Discuss. 11, 335 (1999).

[33] M. A. Hillmyer, W. W. Maurer, T. P. Lodge, F. S. Bates, and K. Almdal, J. Chem. Phys. 103, 4814 (1999).

[34] R. M. Hornreich, M. Luban, and S. Shtrikman, Phys. Rev. Lett. 35, 1678 (1975).

[35] H. W. Diehl, Acta Physica Slovaca 52, 271 (2002).

[36] V. Ganesan, G. H. Fredrickson, Europhys. Lett. 55, 814 (2001).

[37] G. H. Fredrickson, V. Ganesan, F. Drolet, Macromolecules 35, 16 (2002);

[38] G. H. Fredrickson, J. Chem. Phys. 117, 6810 (2002).

[39] T. L. Morkved, P. Stepanek, K. Krishnan, F. S. Bates, and T. P. Lodge, J. Chem. Phys. 114, 7247 (2001).

[40] R. Holyst, M. Schick, J. Chem. Phys. 96, 7728 (1992).

[41] D. Schwahn, K. Mortensen, H. Frielinghaus, K. Almdal, L. Kielhorn, J. Chem. Phys. 112, 5454 (2000).

[42] R. Holyst and Bartosz Przybylski, Phys. Rev. Lett. 85,
130 (2000).

[43] F. S Bates, J. H. Rosedale, G. H. Fredrickson, J. Chem. Phys. 92, 6255 (1990).

[44] H. Fried, K. Binder, J. Chem. Phys. 94, 8349 (1991).

[45] W. W. Maurer, F. S. Bates, T. P. Lodge, K. Almdal, K. Mortensen, and G. H. Fredrickson, J. Chem. Phys. 108, 2989 (1998).

[46] D. Broseta, G. H. Fredrickson, J. Chem. Phys. 93, 2927 (1990).

[47] F. Schmid, M. Schick, Phys. Rev. E 48, 1882 (1993).

[48] M. Teubner and R. Strey, J. Chem. Phys. 87, 3195 (1987).

[49] The attentive reader will notice a certain similarity of some configurations, especially those at higher $\chi N$. The corresponding simulations were indeed started independently of each other, but with the same seed number for the random number generator (which one is allowed to do).

[50] D. C. Morse, Cur. Opin. in Col. \& Int. Sci. 2, 365 (1997). 